\title{
Differential Therapeutic Effects Of Add-on Group v/s Individual Yoga Therapy in Unipolar Depression: A Single Blinded Randomized Controlled Study
}

\author{
Author- Dr. Pratham Dua \\ Co-authors: \\ Dr. Shivarama Varambally, Dr. Naren P Rao, Dr. Urvakhsh M Mehta, Prof. Gangadhar B N \\ NIMHANS, Bengaluru, India
}

\section{BACKGROUND AND AIMS}

Depression is a major neuropsychiatric illness with current estimates exceeding 300 million people worldwide. Pharmacological therapies with antidepressants, psychotherapy or a combination of both are the most commonly used treatments for depression. Both therapies have been shown to be effective for treating depression (Kupfer, 1991). However, recent reviews found high placebo responses and publication bias (Mathew \& Charney, 2009; Pigott, Leventhal, Alter, \& Boren, 2010; Rief et al., 2009; Turner, Matthews, Linardatos, Tell, \& Rosenthal, 2008). Several patients approach complementary and alternative treatment while on medication. In medical literature, one such treatment is Yoga which has been termed as a mind - body medical intervention i.e. an intervention that focuses on interactions among mind, rest of the body and behaviour. Previous systematic reviews have documented a moderate effect size of yoga in treating depression but have not compared the efficacy of individual and group yoga sessions. Moreover, reporting of attrition rates has been inadequate. Therefore, this study aimed to:

- Assess efficacy of individual yoga sessions in comparison to group yoga sessions as an add-on treatment in patients with unipolar depression.

- Assess differential attrition rates between the two yoga arms.

\section{MATERIALS AND METHODS}

34 patients diagnosed with unipolar depression (first or recurrent episode) according to ICD 10 criteria on pharmacotherapy or psychotherapy, or both, were recruited for the study. After written informed consent, 18 (10 males) and 16 (3 males) were randomized into individual and group yoga intervention arms respectively using block randomization in groups of 6 with the primary investigator blinded to randomization. The yoga module developed by Naveen et. al (2013) was followed for intervention. Hamilton Rating Scale for Depression (HAM-D) was administered at baseline and after the end of 12 yoga sessions. Attrition rates and reduction in HAM-D scores within each yoga arm were analyzed and compared postintervention.

Since the sample size was small, non parametric tests were used for statistical analysis. Mann-Whitney $U$ Test was used to compare differential efficacy between the two arms after calculation of change in HAM-D scores pre and post-treatment. Wilcoxon Signed Rank Test was used to analyze improvement in the HAM-D scores within each group (pre and post-treatment).

\section{RESULTS}

The median pre-treatment and post-treatment HAM-D scores for the patients who completed 12 sessions of yoga in individual yoga arm $(n=12)$ was 21 (range: $32-14$ ) and 6.5 (range: 22-0) respectively. The change in the medians and ranges between pre and posttreatment scores was found to be statistically significant ( $V=78$; $\left.p=0.0025^{\star}\right)$.
Similarly, the median pre-treatment and post-treatment HAM-D score for the patients who completed 12 sessions of yoga in the group yoga arm $(n=6)$ was 21.5 (range: $29-14$ ) and 9.5 (range: 23-5) respectively. This change in medians and ranges between pre and post-treatment scores was also statistically significant. $\left(\mathrm{V}=21 ; p=0.031^{\star}\right)$.

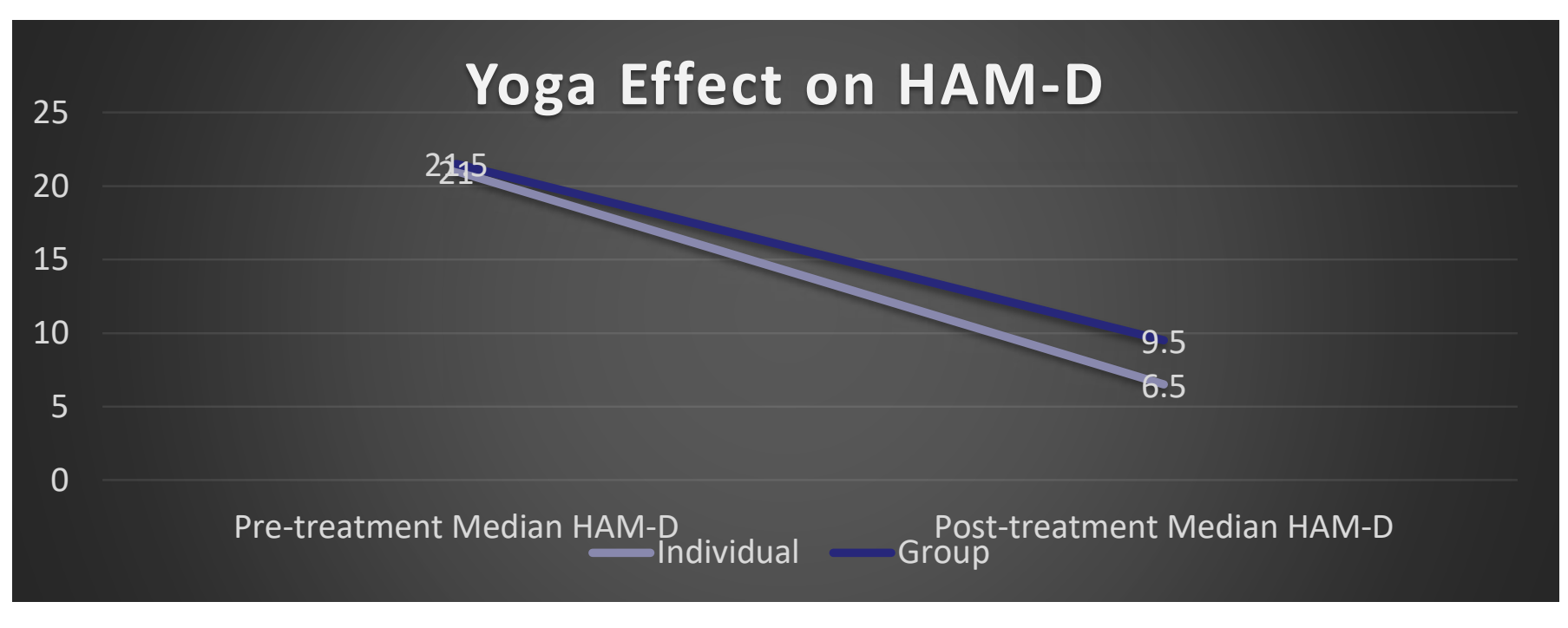

The median change in post-treatment HAM-D scores from pre-treatment scores for the patients who completed 12 yoga sessions in individual yoga arm ( $n=12$ ) was 14 (range: $27-3$ ) and for the group yoga arm ( $n=6)$ was 10 (range: 19-4). There was no statistically significant difference in change in HAM-D scores between the two intervention arms $(U=43$; $p=0.541$ ).

\begin{tabular}{|c|c|c|c|c|c|c|c|c|c|c|}
\hline $\begin{array}{c}\text { Intervention } \\
\text { arm }\end{array}$ & \multicolumn{2}{|c|}{$\begin{array}{c}\text { Patients } \\
\text { recruited } \\
(\mathrm{N}=\mathbf{3 4})\end{array}$} & \multicolumn{2}{|c|}{$\begin{array}{c}\text { Completed } \\
\qquad(\mathrm{N}=18)\end{array}$} & \multicolumn{2}{|c|}{$\begin{array}{l}\text { Dropout } \\
(\mathrm{N}=16)\end{array}$} & \multicolumn{2}{|c|}{$\begin{array}{c}\text { Attrition rate } \\
\text { for gender } \\
(\%)\end{array}$} & \multirow{2}{*}{$\begin{array}{c}\text { Attrition } \\
\text { rate for } \\
\text { intervention } \\
\text { (\%) }\end{array}$} & \multirow[t]{2}{*}{$\begin{array}{l}\text { Overall } \\
\text { attrition } \\
\text { rate }(\%)\end{array}$} \\
\hline Gender & M & F & M & $\mathrm{F}$ & M & F & M & F & & \\
\hline Individual & 10 & 8 & 7 & 5 & 3 & 3 & $30 \%$ & $37.5 \%$ & $33.33 \%$ & $47.05 \%$ \\
\hline Group & 3 & 13 & 0 & 6 & 3 & 1 & $100 \%$ & $53.8 \%$ & $62.5 \%$ & \\
\hline
\end{tabular}

Gender wise attrition rate was noted to be $30 \%$ and $37.5 \%$ for males and females respectively in the individual yoga condition whereas, it was found to be $100 \%$ and $53.8 \%$ for males and females respectively in the group yoga condition. Hence, the total attrition rate for males being $46.15 \%$ and for females being $47.61 \%$ were found to be comparable. Based on the intervention, individual yoga arm had a $33.33 \%$ attrition rate whereas the group yoga arm had an attrition rate of $62.5 \%$, with combined attrition rate for both arms being $47.05 \%$ for this study.

\section{CONCLUSION}

Yoga was effective in reducing depression scores both as an individual and group intervention, although the findings in the study should be cautiously interpreted due to the limited sample size and high attrition rate. There was a high attrition rate, particularly in the group yoga sessions (more in males). This may be possibly due to the difficulty in attending the group yoga sessions which were inflexible and held only once a day during working hours. On the other hand, attrition rate was lower in individual yoga sessions possibly due to individual attention from therapists and flexibility of session timings.

Yet, demonstration of comparable effectiveness for group yoga and individual yoga sessions may favour its use in resource-poor settings. High attrition rates especially in group yoga may indicate the need for innovative and flexible delivery services. 